\title{
IGF1 potentiates BMP9-induced osteogenic differentiation in mesenchymal stem cells through the enhancement of $\mathrm{BMP} /$ Smad signaling
}

\author{
Liang Chen, Xiang Zou, Ran-Xi Zhang, Chang-Jun Pi, Nian Wu, Liang-Jun Yin \& Zhong-Liang Deng* \\ Department of Orthopaedics, The Second Affiliated Hospital, Chongqing Medical University, Chongqing 400010, China
}

\begin{abstract}
Engineered bone tissue is thought to be the ideal alternative for bone grafts in the treatment of related bone diseases. BMP9 has been demonstrated as one of the most osteogenic factors, and enhancement of BMP9-induced osteogenesis will greatly accelerate the development of bone tissue engineering. Here, we investigated the effect of insulin-like growth factor 1 (IGF1) on BMP9-induced osteogenic differentiation, and unveiled a possible molecular mechanism underling this process. We found that IGF1 and BMP9 are both detectable in mesenchymal stem cells (MSCs). Exogenous expression of IGF1 potentiates BMP9-induced alkaline phosphatase (ALP), matrix mineralization, and ectopic bone formation. Similarly, IGF1 enhances BMP9-induced endochondral ossification. Mechanistically, we found that IGF1 increases BMP9-induced activation of BMP/Smad signaling in MSCs. Our findings demonstrate that IGF1 can enhance BMP9-induced osteogenic differentiation in MSCs, and that this effect may be mediated by the enhancement of the $\mathrm{BMP} / \mathrm{Smad}$ signaling transduction triggered by BMP9. [BMB Reports 2016; 49(2): 122-127]
\end{abstract}

\section{INTRODUCTION}

Bone defects and non-union are very common diseases. Bone grafting is currently the main treatment for these diseases, although bone tissue engineering is the best alternative (1). One critical component of bone tissue engineering is growth factors, which should effectively promote differentiation of the seed cells towards an osteoblast lineage (2). The differentiation of precursor cells to osteoblasts is a well-regulated process, in which groups of factors interact with each other at critical

${ }^{*}$ Corresponding author. Tel: +86-13608367586; Fax: +86-2363693609; E-mail: Dengzhongliang7586@126.com

http://dx.doi.org/10.5483/BMBRep.2016.49.2.228

Received 4 November 2015, Revised 19 November 2015, Accepted 8 December 2015

Keywords: BMP9, BMPs/Smads, IGF1, MSCs, Osteogenic differentiation points to direct physiological bone formation. Among these factors, bone morphogenetic proteins (BMPs) are excellent candidates for study $(3,4)$. BMPs belong to the TGF- $\beta$ superfamily, and their osteogenic activities have been demonstrated in numerous preclinical and clinical studies (4). In clinical trials, BMP2 and BMP7 have been evaluated for the treatment of tibia fractures and spine fusion $(5,6)$. BMP9 is the most potent osteogenic BMP, however, the mechanism underlying this process remains unclear. The exploration of novel factors to potentiate BMP9-induced osteogenic differentiation will remarkably accelerate the development of bone tissue engineering.

It is conceivable that certain other factors are able to promote BMP9-induced osteogenesis. Indeed, a small number of factors and signaling pathways have been reported to be associated with the enhancement of BMP9-induced osteogenic differentiation in MSCs, such as Wnt/ $\beta$-catenin (7), retinoic acids signaling (8), and COX-2 (9). Insulin-like growth factors (IGFs) are composed of two subtypes, IGF1 and IGF2. IGFs are wellknown to regulate the proliferation and differentiation of muscle, cartilage, chondrocytes, and neurons (10-12). Moreover, IGFs have been shown to function cooperatively with BMP2 and/or BMP7 to regulate the osteogenic differentiation process $(13,14)$. This evidence suggests that IGFs may also have the potential to promote BMP9-induced osteogenesis in MSCs. IGF1 is secreted primarily in the liver in response to human growth hormone $(\mathrm{GH})$ and acts systemically (15). However, IGF1 can also be found in osteocytes, where it regulates bone turnover during development (16). Although IGF1 can act synergistically with several BMPs in chondrocytes and preosteoblasts $(13,17)$, it remains unclear whether this effect could extend to BMP9-induced osteoblastic differentiation in MSCs.

In this investigation, we introduced a series of in vitro and in vivo experiments to study the effect of IGF1 on BMP9-induced osteogenic differentiation in MSCs, and unveiled a possible molecular mechanism underlying this effect. Our findings may provide another potential and efficacious strategy to enhance BMP9-induced osteogenesis in MSCs, which will greatly accelerate the development of bone tissue engineering. 


\section{RESULTS}

\section{IGF1 and BMP9 are detectable in MSCs, and BMP9 upregulates the expression of IGF1}

Firstly, we examined the endogenous expression of IGF1 in MSCs using RT-PCR and Western blotting. We found that IGF1 was detectable in $\mathrm{C} 3 \mathrm{H} 10 \mathrm{~T} 1 / 2, \mathrm{MEF}$, and $\mathrm{C} 2 \mathrm{C} 12$ cells (Fig. 1A and B), as is BMP9. To study the effect of IGF1 on BMP9-induced osteogenesis, we constructed recombinant adenoviruses for IGF1 and BMP9. Western blotting analysis showed that the respective recombinant adenoviruses greatly enhanced the expression of IGF1 (Fig. 1D) and BMP9 (Fig. 1E). Since C3H10T1/2 cells are one of the most commonly used progenitor cells for osteogenic differentiation research, this is the cell line we employed in the current investigation. Western blotting analysis showed that BMP9 markedly upregulated IGF1 in C3H10T1/2 cells (Fig. 1G), as it can IGF2. These data suggest that BMP9 and IGF1 are both present in MSCs, and that IGF1 may be associated with BMP9-induced osteogenesis in MSCs.
A

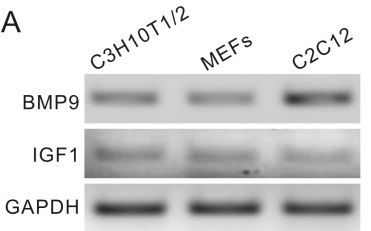

C
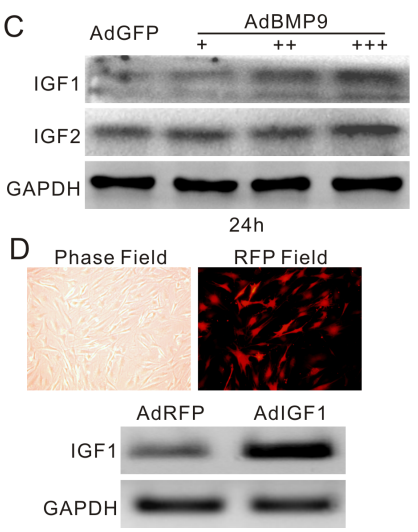
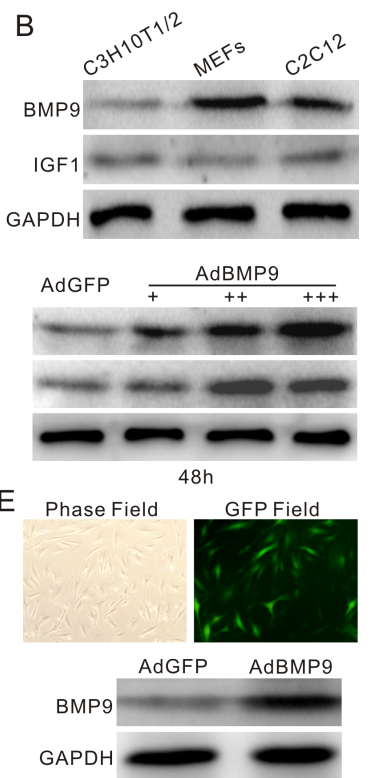

Fig. 1. Endogenous expression of BMP9 and IGF1 in progenitor cells and BMP9-induced expression of IGF1 in C3H10T1/2 cells. (A) PCR results show the endogenous expression of BMP9 and IGF1 in the three progenitor cells. GAPDH was used as a loading control. (B) Western blots show the endogenous expression of BMP9 and IGF1 in the three progenitor cells. GAPDH was used as a loading control. (C) Western blots show the BMP9-induced expression of IGF1 and IGF2 in C3H10T1/2 cells. GAPDH was used as a loading control. (D) Representative image and Western blot show the expression of IGF1 mediated by the adenovirus. GAPDH was used as loading control. (E) Representative image and Western blot show the expression of BMP9 mediated by the adenovirus. GAPDH was used as a loading control.

\section{Exogenous expression of IGF1 potentiates BMP9-induced ALP activity, osteopontin, and matrix mineralization in C3H10T1/2 cells}

Next, we tested whether IGF1 could affect BMP9-induced osteogenic differentiation in MSCs. Subconfluent C3H10T1/2 cells were infected with AdGFP, AdBMP9, and/or AdIGF1. We found that exogenous expression of IGF1 exhibited no significant effect on ALP activity; BMP9 increased ALP activity in C3H10T1/2 cells, which could be strikingly enhanced when combined with IGF1 (Fig. 2A and B). Similar results were found with the expression of osteopontin (OPN) (Fig. 2C) on day 9. Alizarin Red S staining showed that the mineralized matrix nodules were readily detected in the BMP9-treated group, and the mineralized nodules in the BMP9-combined with IGF1-treated group were more pronounced than those in the group treated with BMP9 or IGF1 only (Fig. 2D). These data suggest that IGF1 may potentiate BMP9-induced osteogenic differentiation in MSCs, although IGF1 alone exerts no substantial osteogenic effects.

IGF1 potentiates BMP9-induced ectopic bone formation Next, we tested the effect of IGF1 on BMP9-induced osteogenesis in vivo. C3H10T1/2 cells were infected with AdRFP, AdBMP9, AdIGF1, or AdBMP9 and AdIGF1, collected, and subcutaneously injected into the flank of athymic nude mice. 5 weeks post-injection, the mice were sacrificed and the bone masses were retrieved. No bone masses were found in the AdRFP- or AdIGF1-treated groups. As a general observation, the volume of bone masses from the AdBMP9 combined with
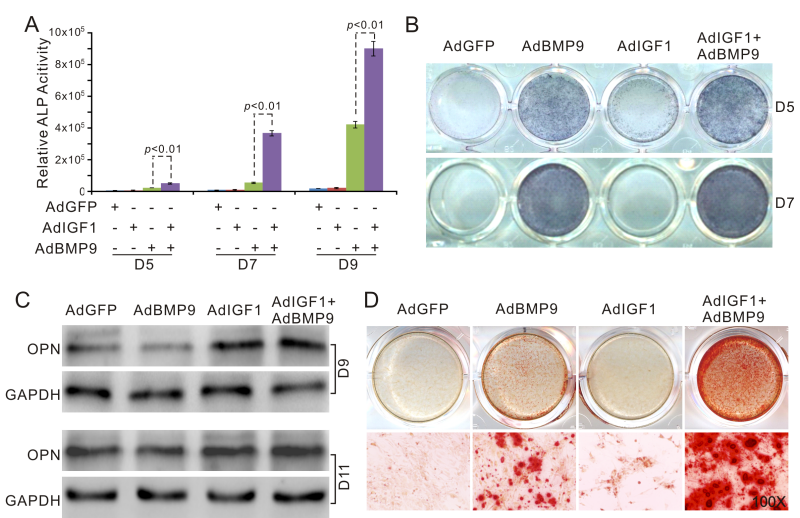

Fig. 2. Effect of IGF1 on different BMP9-induced osteogenic markers in C3H10T1/2 cells. (A) ALP activity shows the effect of IGF1 on BMP9-induced osteogenic differentiation in C3H10T1/2 cells. (B) ALP staining shows the effect of IGF1 on BMP9-induced osteogenic differentiation in $\mathrm{C} 3 \mathrm{H} 10 \mathrm{~T} 1 / 2$ cells. (C) Western blots show the effect of IGF1 on the expression of BMP9-induced OPN in $\mathrm{C} 3 \mathrm{H} 10 \mathrm{~T} 1 / 2$ cells. GAPDH was used as a loading control. (D) Alizarin Red S staining shows the effect of IGF1 on BMP9-induced mineralization in $\mathrm{C} 3 \mathrm{H} 10 \mathrm{~T} 1 / 2$ cells on day 20. The upper panel shows the scanning results, and the lower panel shows the images taken with the microscope. 


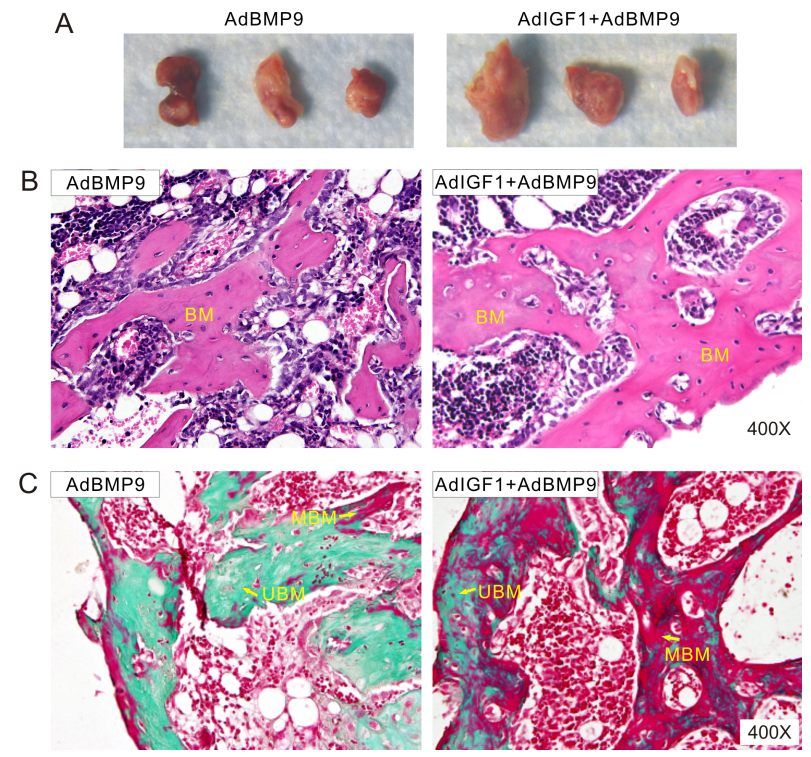

Fig. 3. Effect of IGF1 on BMP9-induced ectopic bone formation. (A) Representative retrieved bone masses show the effect of IGF1 on BMP9-induced ectopic bone formation. (B) H\&E staining shows the effect of IGF1 on BMP9-induced ectopic bone formation. Representative images are shown (BM, bone matrix). (C) Masson's Trichrome staining shows the effect of IGF1 on BMP9-induced ectopic bone formation. Representative images are shown (MBM, matured bone matrix; UBM, unmatured bone matrix).

AdIGF1 group were larger than those from the group treated with AdBMP9 only (Fig. 3A). H\&E staining showed that a greater amount and thicker trabecular bone was present in the AdBMP9-combined with AdIGF1-treated group compared with the group treated with AdBMP9 only (Fig. 3B). Masson's trichrome staining showed that IGF1 promoted the maturation of BMP9-induced bone matrix (Fig. 3C). These data strongly suggest that IGF1 may be a strong enhancer for BMP9 in the induction of osteogenesis in MSCs.

\section{IGF1 enhances BMP9-activated BMP/Smad signaling transduction in $\mathrm{C} 3 \mathrm{H} 10 \mathrm{~T} 1 / 2$ cells}

BMPs usually exert their physiological functions through BMP/Smad signaling, thus, we next investigated whether IGF1 could affect the BMP9-activated BMP/Smad signaling transduction in MSCs. The results showed that BMP9 alone could effectively promote the transcriptional activity of this BMP/ Smad reporter, and that IGF1 alone could also moderately increase this activity. However, the transcriptional activity of this reporter was prominently enhanced when BMP9 was combined with IGF1 (Fig. 4A). Immunocytochemical staining and Western blotting showed that IGF1 could promote the BMP9induced phosphorylation of Smad1/5/8 in C3H10T1/2 cells (Fig. 4B, C and D). These findings indicate that IGF1 may potentiate BMP9-induced activation of BMP/Smad signaling.
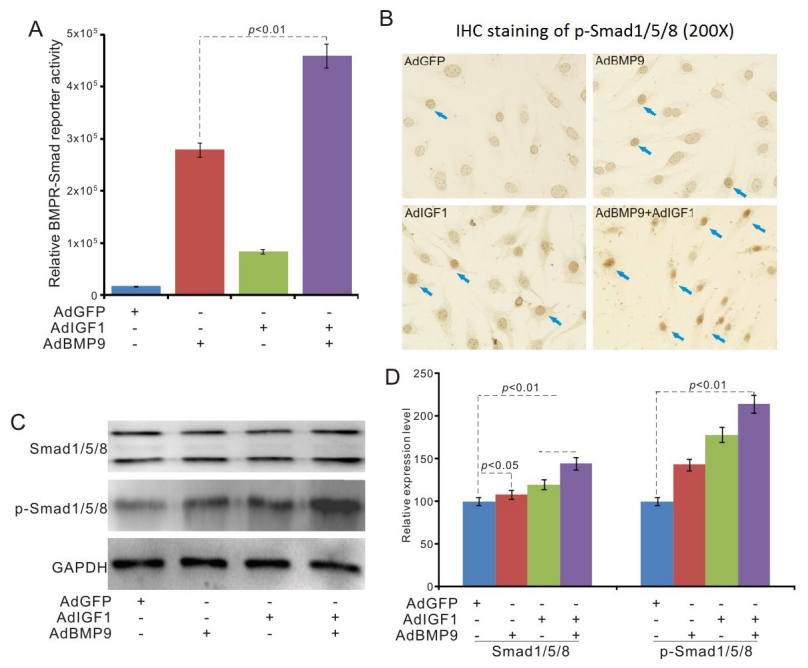

Fig. 4. Effect of IGF1 on BMP9-induced activation of BMP/Smad signaling in $\mathrm{C} 3 \mathrm{H} 10 \mathrm{~T} 1 / 2$ cells. (A) Luciferase reporter activity shows IGF1 potentiates the activation of BMP9-induced BMP/Smad signaling. (B) Immunocytochemical staining shows the effect of IGF1 on BMP9-induced Smad1/5/8 phosphorylation (blue arrow shows the positive staining of Phosphorylated Smad1/5/8 in C3H10T1/2 cells). (C) Western blots show the effect of IGF1 on the level of BMP9-induced Smad1/5/8 and phosphorylation of Smad1/5/8. GAPDH was used as a loading control. (D) Quantification of Western blots shows the effect of IGF1 on the level of BMP9-induced Smad1/5/8 and phosphorylation of Smad1/5/8.

\section{DISCUSSION}

BMP9, as one of the most potent osteogenic factors, may be widely used in bone tissue engineering for the treatment of bone defects, non-union, and fractures (4). In this investigation, we studied the effect of IGF1 on BMP9-induced osteogenic differentiation in MSCs, and found that IGF1 could substantially enhance BMP9-induced osteogenic differentiation. Mechanistically, we demonstrated that the effect of IGF1 on the promotion of BMP9-induced osteogenic differentiation may be mediated by the enhancement of BMP9-activated $\mathrm{BMP} / \mathrm{Smad}$ signaling transduction in MSCs.

BMPs belong to the TGF- $\beta$ superfamily, of which there are approximately 20 members. Several BMPs such as BMP2 and BMP7 can induce MSCs to undergo osteogenic differentiation (18), with BMP9 being reported as the most potent osteogenic BMP to date (4). BMPs usually exert their physiological functions through the BMP/Smad pathway (19). Aside from this pathway, certain other essential factors or signaling pathways such as COX-2 (9), Wnt/ $\beta$-catenin $(7,20)$, retinoic acids signaling $(8,20)$, and IGF2 $(21)$ regulate or potentiate the osteogenic activity of BMP9 in MSCs.

The IGF system comprises two ligands (IGF1 and IGF2), two receptors (IGF1R and IGF2R), seven binding proteins (IGF binding proteins, IGFBP1 - IGFBP7) with high affinity for IGFs, 
and IGFBPs degrading enzymes. IGF signaling is critical for the regulation of development and homeostasis, including differentiation, proliferation, and apoptosis (10-12, 22). In the skeletal system, IGFs are involved in the regulation of bone development, repair, and regeneration. Our previous study demonstrated that IGF2 could promote BMP9-induced osteogenesis (21), thus, theoretically, IGF2 may be used as a potent factor combined with BMP9 to induce osteogenesis in bone tissue engineering. However, IGF2 is expressed mainly during embryonic development, and is thought of as a major fetal growth factor (23). After birth, IGF2 remains at very low levels, and IGF2 overexpression may cause tissue hyperplasia $(23,24)$. For this reason, the combination of IGF2 and BMP9 may be high risk in clinical applications. In contrast, IGF1 has shown a different phenotype, being constantly secreted in response to growth hormones in the liver, and released systemically after birth $(25,26)$. IGF1 functions as a major growth factor in adults and is well-known to have anabolic effects on a wide variety of tissues including muscle, cartilage, and bone (22, 27-29). IGF1 null mice are born at approximately $60 \%$ birth weight compared to their littermates, but have longer life spans $(29,30)$. In the skeletal system, deletion or functional loss of IGF1 may contribute to age-related osteopenia (15). The level of IGF1 increases notably during bone regeneration or repair (31). When combined with VEGF, IGF1 can decoy progenitor cells towards osteogenic differentiation (32). This evidence implies that IGF1 may be more important and safe in the regulation of bone metabolism than IGF2. Although BMP9 was first found in hepatocytes, where IGF1 is also secreted, the effect of IGF1 on BMP9-induced osteogenic differentiation remains unknown.

Our findings suggest that IGF1 may potentiate BMP9-induced osteogenic differentiation in MSCs. Although IGF1 and BMP9 are both present in progenitor cells, osteogenic differentiation does not occur at this stage, which may be due to the fact that the endogenous levels of these two factors are far below the minimal levels needed to initialize osteogenic commitment. Using recombinant adenoviruses, we found that IGF1 enhanced BMP9-induced ALP activity and matrix mineralization in $\mathrm{C} 3 \mathrm{H} 10 \mathrm{~T} 1 / 2$ cells (Fig. 2A-D). The results of the in vivo experiments showed that the bone volume and maturity of the BMP9-combined with IGF1-treated group was greater than that of the groups treated with IGF1 or BMP9 alone (Fig. 3B and C). $\mathrm{BMP} / \mathrm{Smad}$ is the classical signaling pathway that mediates the osteogenic activity of BMP9 $(33,34)$. Our data indicates that IGF1 can enhance the activation of BMP9-induced BMP/Smad signaling in MSCs (Fig. 4A-D). All these data support the idea that IGF1 can potentiate BMP9-induced osteogenic differentiation in MSCs.

Taken together, the findings of this investigation strongly suggest that IGF1 is an excellent candidate enhancer for BMP9-induced osteogenic differentiation in MSCs, which may be mediated by the promotion of BMP9-initialized activation of BMP/Smad signaling transduction.

\section{MATERIALS AND METHODS}

\section{Cell culture and chemicals}

HEK293, C3H10T1/2, and C2C12 cells were purchased from ATCC (VA, USA). Cells were maintained in Dulbecco's modified Eagle's medium (DMEM) with 10\% fetal bovine serum (FBS), $100 \mathrm{U} / \mathrm{ml}$ penicillin, and $100 \mu \mathrm{g} / \mathrm{ml}$ streptomycin at $37^{\circ} \mathrm{C}$ in $5 \% \mathrm{CO}_{2}$. All antibodies were purchased from Santa Cruz Biotechnology (CA, USA), and the PI3K inhibitor, LY294002, was bought from Selleck Chemicals (TX, USA).

\section{Construction of recombinant adenoviruses expressing RFP, GFP, BMP9, and IGF1}

Recombinant adenoviruses were generated using AdEasy technology as previously described (35). The coding sequences of human BMP9 and IGF1 were amplified using PCR, cloned into an adenoviral shuttle vector, and subsequently used to generate recombinant adenoviruses in HEK293 cells. The recombinant adenoviruses were designated as AdBMP9 and AdIGF. AdBMP9 was tagged with green fluorescent protein (GFP) and AdIGF1 was tagged with red fluorescent protein (RFP), as markers to track the viruses. Analogous adenovirus expressing only monomeric RFP (AdRFP) or GFP (AdGFP) were used as controls.

\section{Isolation of mouse embryonic fibroblasts (MEFs)}

MEFs were isolated from mice on post-coitus day 13.5 as previously described $(3,8,9)$. Each embryo was dissected into 10 $\mathrm{ml}$ sterile PBS, voided of its internal organs, and sheared through an 18-gauge syringe in the presence of $1 \mathrm{ml} 0.25 \%$ trypsin and $1 \mathrm{mM}$ EDTA. Following a 15-minute incubation with gentle shaking at $37^{\circ} \mathrm{C}$, DMEM with $10 \%$ FBS was added to inactivate the trypsin. The cells were plated on $100-\mathrm{mm}$ dishes and incubated for 24 hours at $37^{\circ} \mathrm{C}$, following which adherent cells were used as MEF cells. Aliquots were kept in liquid nitrogen. MEFs used in this study were within 5 passages.

\section{RNA extraction and semi-quantitative RT-PCR analysis}

Total RNA was isolated using TRIZOL reagents (Invitrogen), and used to generate cDNA templates by a RT reaction with hexamer and Superscript II RT (Invitrogen). The first strand CDNA products were further diluted 5-10 fold and used as PCR templates. Semi-quantitative RT-PCR was carried out as described. PCR primers were designed using the Primer3 program in order to detect the expression of genes of interest. All samples were normalized to the expression of GAPDH.

\section{Alkaline phosphatase (ALP) assay}

ALP activity was assessed using a modified Great Escape SEAP chemiluminescence assay (BD Clontech, Mountain View, CA) and/or histochemical staining as described previously $(4,7,9$, 21). Cells were treated with the corresponding reagents as per the protocols. Each assay was performed in triplicate, and re- 
peated in at least three independent experiments. ALP activity was normalized to the level of total cellular protein.

\section{Transfection and luciferase reporter assay}

Exponentially growing cells were seeded in T25 flasks and transfected with $2 \mu \mathrm{g} /$ flask BMPR-Smad responsive element luciferase reporter (p12xSBE-Luc) (36), using Lipofectamine (Invitrogen). 16 hours post-transfection, cells were replated on 24-well plates and infected with AdBMP9, AdIGF1, and/or AdRFP at 4 hours after replating. 24 hours post-infection, cells were lysed and the lysates were collected for luciferase assays using a kit (Promega). Each assay condition was performed in triplicate.

\section{Matrix mineralization assay (Alizarin Red S staining)}

Cells were seeded on 24-well cell culture plates and infected with AdGFP, AdBMP9, and/or AdIGF1. Cells were cultured in the presence of ascorbic acid $(50 \mu \mathrm{g} / \mathrm{ml})$ and $\beta$-glycerophosphate $(10 \mathrm{mM}) .20$ days post-infection, mineralized matrix nodules were stained with Alizarin Red $\mathrm{S}$ as described previously $(4,7,20,21)$. Cells were fixed with $0.05 \%(v / v)$ glutaraldehyde at room temperature for 10 minutes. After washing with distilled water, fixed cells were incubated with $0.4 \%$ Alizarin Red S (Sigma-Aldrich) for 5 minutes, followed by extensive washing with distilled water. The staining of mineralized matrix nodules was scanned and/or imaged under a microscope.

\section{Immunocytochemical staining}

Cultured cells were infected with the corresponding adenoviruses. Cells were fixed with $10 \%$ formalin and washed with PBS at the indicated time points. The fixed cells were permeabilized with $1 \%$ NP-40 and blocked with $10 \%$ goat serum, followed by incubation with a $\mathrm{p}-\mathrm{Smad} 1 / 5 / 8$ primary antibody for 1 hour. Cells were then incubated with a biotin-labeled secondary antibody for 25 minutes, followed by incubation with streptavidin-HRP for 20 minutes at room temperature. The target protein was visualized by DAB staining and imaged under a microscope. Staining with the corresponding $\lg G$ was used as a negative control.

\section{Ectopic bone formation}

Cells were infected with adenoviruses as previously described $(4,8,9,20,21)$. The animal experiment was approved by the Institutional Animal Care and Use Committee (IACUC) of Chongqing Medical University. 16 hours post-infection, cells were harvested and resuspended in PBS for subcutaneous injection ( $5 \times 10^{6}$ /injection) to the flanks of athymic nude mice ( 5 mice per group, 4-6 weeks old, female). 5 weeks post-injection, mice were sacrificed and the bone masses were retrieved for histological evaluation.

\section{Histological evaluation and trichrome staining}

Retrieved bone masses were fixed in $10 \%$ formalin (decalcified if necessary) and embedded in paraffin. Paraffin-embedded sections were deparaffinized and then rehydrated in a graduated fashion. Deparaffinized sections were subjected to antigen retrieval and fixation, and then to hematoxylin and eosin (H\&E) or Masson's Trichrome staining.

\section{Statistical analysis}

The results of all experiments are expressed as the mean \pm standard deviation (SD) of at least three independent experiments. A Student's t-test was used for single-variable comparisons, and a $\mathrm{P}$ value $<0.05$ was considered statistically significant. Each assay condition was performed in triplicate, and the results were repeated in at least three independent experiments.

\section{ACKNOWLEDGEMENTS}

The authors wish to thank Dr. Di Chen of Rush University Medical Center for providing the p12xSBE-Luc plasmid. This work was supported by research grants from the Natural Science Foundation of China and Chongqing Science and Technology Commission (NSFC, 81272005; cstc2013jjB10021 to Zhong-liang Deng; cstc2015jcyjA10087 to Liang Chen).

\section{REFERENCES}

1. Harada N, Watanabe $Y$, Sato $K$ et al (2014) Bone regeneration in a massive rat femur defect through endochondral ossification achieved with chondrogenically differentiated MSCs in a degradable scaffold. Biomaterials 35, 7800-7810

2. Amini AR, Laurencin CT and Nukavarapu SP (2012) Bone tissue engineering: recent advances and challenges. Crit Rev Biomed Eng 40, 363-408

3. Huang J, Yuan SX, Wang DX et al (2014) The role of COX-2 in mediating the effect of PTEN on BMP9 induced osteogenic differentiation in mouse embryonic fibroblasts. Biomaterials 35, 9649-9659

4. Kang Q, Sun $\mathrm{MH}$, Cheng $\mathrm{H}$ et al (2004) Characterization of the distinct orthotopic bone-forming activity of 14 BMPs using recombinant adenovirus-mediated gene delivery. Gene Ther 11, 1312-1320

5. Khosla S, Westendorf JJ and Oursler MJ (2008) Building bone to reverse osteoporosis and repair fractures. J Clin Invest 118, 421-428

6. Cooper GS and Kou TD (2013) Risk of cancer after lumbar fusion surgery with recombinant human bone morphogenic protein-2 (rh-BMP-2). Spine (Phila Pa 1976) 38, 1862-1868

7. Zhang H, Wang J, Deng F et al (2014) Canonical Wnt signaling acts synergistically on BMP9-induced osteo/odontoblastic differentiation of stem cells of dental apical papilla (SCAPs). Biomaterials 39C, 145-154

8. Zhang W, Deng ZL, Chen L et al (2010) Retinoic acids potentiate BMP9-induced osteogenic differentiation of mesenchymal progenitor cells. PLoS One 5, e11917

9. Wang JH, Liu YZ, Yin LJ et al (2013) BMP9 and COX-2 
form an important regulatory loop in BMP9-induced osteogenic differentiation of mesenchymal stem cells. Bone 57, 311-321

10. Sullivan KA, Kim B and Feldman EL (2008) Insulin-like growth factors in the peripheral nervous system. Endocrinology 149, 5963-5971

11. Patil AS, Sable RB and Kothari RM (2012) Role of insulin-like growth factors (IGFs), their receptors and genetic regulation in the chondrogenesis and growth of the mandibular condylar cartilage. J Cell Physiol 227, 1796-1804

12. Wang L, Han Y, Shen Y et al (2014) Endothelial insulinlike growth factor-1 modulates proliferation and phenotype of smooth muscle cells induced by low shear stress. Ann Biomed Eng 42, 776-786

13. Kim S, Kang Y, Krueger CA et al (2012) Sequential delivery of BMP-2 and IGF-1 using a chitosan gel with gelatin microspheres enhances early osteoblastic differentiation. Acta Biomater 8, 1768-1777

14. Joung YH, Lim EJ, Darvin P et al (2013) Hwanggeumchal sorghum extract enhances BMP7 and $\mathrm{GH}$ signaling through the activation of Jak2/STAT5B in MC3T3E1 osteoblastic cells. Mol Med Rep 8, 891-896

15. Perrini S, Laviola L, Carreira MC, Cignarelli A, Natalicchio A and Giorgino F (2010) The GH/IGF1 axis and signaling pathways in the muscle and bone: mechanisms underlying age-related skeletal muscle wasting and osteoporosis. J Endocrinol 205, 201-210

16. Sheng MH, Zhou XD, Bonewald LF, Baylink DJ and Lau KH (2013) Disruption of the insulin-like growth factor-1 gene in osteocytes impairs developmental bone growth in mice. Bone 52, 133-144

17. Yoon DM and Fisher JP (2006) Chondrocyte signaling and artificial matrices for articular cartilage engineering. Adv Exp Med Biol 585, 67-86

18. Chen D, Zhao M and Mundy GR (2004) Bone morphogenetic proteins. Growth Factors 22, 233-241

19. Lamplot JD, Qin J, Nan G et al (2013) BMP9 signaling in stem cell differentiation and osteogenesis. Am J Stem Cells 2, 1-21

20. Liu Y, Liu Y, Zhang R et al (2014) All-trans retinoic acid modulates bone morphogenic protein 9-induced osteogenesis and adipogenesis of preadipocytes through BMP/ Smad and Wnt/beta-catenin signaling pathways. Int J Biochem Cell Biol 47, 47-56

21. Chen L, Jiang W, Huang J et al (2010) Insulin-like growth factor 2 (IGF-2) potentiates BMP-9-induced osteogenic differentiation and bone formation. J Bone Miner Res 25, 2447-2459

22. Huat TJ, Khan AA, Pati S, Mustafa Z, Abdullah JM and Jaafar H (2014) IGF-1 enhances cell proliferation and sur- vival during early differentiation of mesenchymal stem cells to neural progenitor-like cells. BMC Neurosci 15, 91

23. Livingstone C (2013) IGF2 and cancer. Endocr Relat Cancer 20, R321-339

24. Brouwer-Visser J and Huang GS (2015) IGF2 signaling and regulation in cancer. Cytokine Growth Factor Rev 26, 371-377

25. Svensson J, Tivesten A, Sjogren K et al (2007) Liver-derived IGF-I regulates kidney size, sodium reabsorption, and renal IGF-II expression. J Endocrinol 193, 359-366

26. Sjogren K, Sheng M, Moverare S et al (2002) Effects of liver-derived insulin-like growth factor I on bone metabolism in mice. J Bone Miner Res 17, 1977-1987

27. Tirapegui J (1999) Effect of insulin-like growth factor-1 (IGF-1) on muscle and bone growth in experimental models. Int J Food Sci Nutr 50, 231-236

28. Schlegel W, Raimann A, Halbauer D et al (2013) Insulinlike growth factor I (IGF-1) Ec/Mechano Growth factor-a splice variant of IGF-1 within the growth plate. PLoS One 8, e76133

29. Brown-Borg HM and Bartke A (2012) GH and IGF1: roles in energy metabolism of long-living $\mathrm{GH}$ mutant mice. J Gerontol A Biol Sci Med Sci 67, 652-660

30. Junnila RK, List EO, Berryman DE, Murrey JW and Kopchick JJ (2013) The GH/IGF-1 axis in ageing and longevity. Nat Rev Endocrinol 9, 366-376

31. Sheng MH, Lau KH and Baylink DJ (2014) Role of Osteocyte-derived Insulin-Like Growth Factor I in Developmental Growth, Modeling, Remodeling, and Regeneration of the Bone. J Bone Metab 21, 41-54

32. Ferretti C, Vozzi G, Falconi M et al (2014) Role of IGF1 and IGF1/VEGF on human mesenchymal stromal cells in bone healing: two sources and two fates. Tissue Eng Part A 20, 2473-2482

33. Retting KN, Song B, Yoon BS and Lyons KM (2009) BMP canonical Smad signaling through Smad1 and Smad5 is required for endochondral bone formation. Development 136, 1093-1104

34. David L, Mallet C, Mazerbourg S, Feige JJ and Bailly S (2007) Identification of BMP9 and BMP10 as functional activators of the orphan activin receptor-like kinase 1 (ALK1) in endothelial cells. Blood 109, 1953-1961

35. Luo J, Deng ZL, Luo X et al (2007) A protocol for rapid generation of recombinant adenoviruses using the AdEasy system. Nat Protoc 2, 1236-1247

36. Zhao M, Qiao M, Oyajobi BO, Mundy GR and Chen D (2003) E3 ubiquitin ligase Smurf1 mediates core-binding factor alpha1/Runx2 degradation and plays a specific role in osteoblast differentiation. J Biol Chem 278, 2793927944 